\title{
A Comprehensive Study of Various Load Balancing Techniques used in Cloud Based Biomedical Services
}

\author{
Abhinav Hans* and Sheetal Kalra \\ GNDU RC Jalandhar \\ abhinavhans@gmail.com
}

\begin{abstract}
With an increase in the demands, the Cloud computing has become one of the ongoing scalable approaches to fulfill the cloud based services. The biggest advantage of the cloud computing is the ability to overcome the infrastructural challenges those are earlier faced by other technologies. Since the technology is new, therefore the development of the whole structure is not so efficient. It does have a lot of issues on which various scientists and others are working on. Scheduling, load balancing, fault tolerance, etc. are various challenges faced by cloud computing. For this purpose various techniques and algorithms have been proposed. In this paper, we will discuss the issue of load balancing of cloud computing and we will study the different types of load balancing techniques used in in biomedical services and make a comparative analysis among all the existing techniques.
\end{abstract}

Keywords: $(O L B+$ LBMM), Genetic Algorithm, VectorDot, Stochastic Hill Climbing, LBVS- H, Ant colony optimization algorithm, Compare and Balance, Honey Bee Foraging

\section{Introduction}

Cloud computing is one the emerging technology, which provides the service of software for lease. Cloud computing allows the user to make demands and provide the services accordingly to the users. People now a day's get the services according to their demands therefore they do not have to pay unnecessary for the things they don't have to use. People now a day's get the services according to their demands therefore they do not have to pay unnecessary for the things they don't have to use. Cloud computing is efficient and scalable, but maintaining the stability of processing so many jobs in the cloud computing environment is a very complex problem with load balancing receiving much attention from researchers [1].

At the infrastructural level, it is impossible to maintain the one or more free service to fulfill the demand at the same time. Since without any load balancing techniques the system will face the traffic and ultimately gets crashed. Application of load balancing and redundant mirrored databases in cluster techniques, across multiple availability zones, reduces the chance of outages that could simultaneously affect the services in cloud systems [2]. We will discuss the various load balancing comparison parameters and then give a brief introduction to all the load balancing techniques existed. And the end of the paper, we will discuss the comparison among all. Recently, public cloud has been made available as a pay per usage model while private cloud can be built with the infrastructure of the organization itself [3]. Scalability, one of the very important features of cloud computing, is also enabled by load balancing [5].

\section{Measurement Parameters for Various Load Balancing Techniques}

An efficient load balancing techniques is the one that avoids the traffic and balances the load equally therefore there must be some parameters to evaluate the load balancing 
techniques to get better resource distribution for the user demands. In this paper, we used various load balancing measurement parameters to evaluate the load balancing techniques which are discussed below:

- Throughput: It is the amount of outputs that a computer can perform on a given input in the given amount of time. execution.

Response time: It is the amount of time used to put the user query in concern for

- Fault tolerance: It is the ability of the load balancing algorithm that allows to keep working properly in any failure condition of the system.

- Scalability: It is the ability to expand itself according to required conditions.

- Performance: It is the overall check of the algorithms working. It comprises the completion of the given task against present known standards like accuracy, cost and speed.

- Resource utilization: It is used to keep a check on the utilization of various resources.

\section{Load Balancing Algorithms}

Various load balancing techniques used in cloud based biomedical servces are discussed below:

A. $(O L B+L B M M):$ In [10] author had proposed a two-stage scheduling algorithm that aggregates OLB (Opportunistic Load Balancing) and LBMM (Load Balance MinMin) scheduling algorithms to employ better accomplishing efficiency and conserve the load balancing of the scheme. In OLB scheduling algorithm, each node is kept in an operative state to attain the destination of load balance and LBMM scheduling algorithm is employed to downplay the execution time of each job on the node thereby downplaying the overall windup time. This aggregated coming hence aids in an efficient employment of resources and raises the job efficiency.

B. Genetic Algorithm: In [7] author proposed a novel algorithm for load balancing using genetic approaches. Author tries to eliminate the challenge of the inappropriate distribution of the execution time, which uses to create the traffic on the server. The Author had used a simulation environment to perform the execution and results of their own proposed algorithm. Also author compared its simulation result with the existing techniques like First come first server (FCFS) and Round Robin (RR).

C. VectorDot: In [8] author had purposed a new load balancing algorithm called VectorDot. VectorDot holds the hierarchical complexes of the data center and multidimensionality of resource piles crosswise servers, network switches, and storage in a nimble data center that has desegregated server and memory virtualization engineering sciences. VectorDot exercises dot product to differentiate nodes based on the item necessities and helps in getting rid of overburdens on servers, switches and storage nodes.

D. Stochastic Hill Climbing: In [11] author proposed a mild figuring load balancing approach for load balancing. Author used the local optimization scheme stochastic hill climbing for allotment of ingress chores to the virtual servers or servers. Author examined the execution of the algorithm with the help of the cloudAnalyst simulator, which is a cloudSim visual modeler. The execution is examined both quantitatively and qualitatively and made a comparison with Round Robin (RR) and First Come First Serve (FCFS).It is one of the kinds of the hill climbing algorithm which is utilized for the load balancing. As like hill climbing, which prefers the most outrageous uphill 
motility stochastic hill climbing opts arbitrarily from the uphill moves with effectual chance.

E. LBVS- H.: In [12] author had proposed a load balancing, virtual depot scheme (LBVS) that offers a large shell net data storage model and Storage as a Service model based on Cloud Storage. Reposition virtualization is accomplished using a scenario that is three-layered and load balancing is attained using two load balancing parts. It aids in bettering the efficiency of coincident admittance by using replication balancing further slimming the response time and raising the content of tragedy recuperation. This strategy also helps in improving the usage pace of storage resource, flexibleness and lustiness of the scheme.

F. Ant colony optimization algorithm: In [4] author had proposed a load balancing algorithm based on trial laying principle. Ants when cracks out for hunt of food riffs a trail of the track for the other ants so that they do not have to recover the track. The ants will just follow the dogged route and will discover food. Likewise, the author used this precept and asserts a pheromone table of the route that the factors use from origin to goal and the routing tastes. The route is updated at each new position with the distance measure which admits the other mobile agents to choose the littlest route. The Author had used this algorithm for load balancing of the requirements on the cloud by discovering the server's free content and processing all the servers as the virtual server. To resolve the trouble of the load balancing author had chosen the demeanor of the ants in hunt of the food. Since ants had a very well-informed way for discovering the food by the method of shortest distance, author took that into the consideration. Ants uses the rule of trail lying by omitting the pheromones on the land by ending on some points on the way through their motion which secreted by pheromone gland. That put a trail for the ants to come back to their dependency after they found food.

G. Compare and Balance: In [13] author had directed the trouble of intra-cloud load balancing amongst strong-arm hosts by adaptive live migration of virtual machines. A load balancing model is planned and carried out to melt off virtual machines' migration time by partook storage, to counterweight load amongst servers according to their processor or IO usage, etc. and to keep virtual machines' zero-downtime in the process. A dispersed load balancing algorithm compare and balance is also proposed that is based on sampling and reaches equilibrium very fast. This algorithm promises that the migration of VMs has been always from high-cost strong-arm hosts to lowcost host, but arrogates that each physical host has enough memory which is a frail supposition.

H. Honey Bee Foraging: In [9] author had proposed an algorithm honey bee foraging algorithm. Honey bee with the dancing deportment instigated the author to use this thought for the algorithm. When honey bees go in hunt of food, they do the exceptional dance known as the waggle dance on obtaining the food to state their leftover members that they had found the food. The character of the dance states the quality and the quantity of the food they found and also the dance tells the accurate outdistance of food from the beehive. Author used this thought and sorted the servers below the virtual server with their own virtual waiting line. Each server treating the requirement from its queue first computes the lucre which is corresponding to the lineament that bees display in waggle dance. In load balancing this gain or the waggle dance is matched to the measure of time needful to meet the petition or the resources used to satisfy the postulation. 


\section{Conclusion and Future Work}

Cloud computing is more usable now a day's therefore the load balancing is becoming a big challenge to overcome. There are various techniques that are proposed by the various researchers to overcome the challenge of load balancing. In this paper we have made a survey and compared various existing load balancing techniques used in biomedical services. We have made the comparative analysis of different algorithms of load balancing using certain parameters. Since all the techniques covered in this paper are not fully effective, therefore there must develop new techniques which can overcome the parameters like fault tolerance and response time. There are many more parameters which can affect the efficiency of the load balancing techniques which can be included in further study. Therefore, to propose a new technology, one must include the new parameters to evaluate the results.

\section{Comparision of Existing Load Balancing Techniques Based on Measurement Parameters Discussed in Section 2}

\begin{tabular}{|c|c|c|c|c|c|c|c|}
\hline Metrics & Throughput & $\begin{array}{c}\text { Response } \\
\text { time }\end{array}$ & $\begin{array}{c}\text { Fault } \\
\text { tolerance }\end{array}$ & Scalability & Performance & $\begin{array}{c}\text { Resource } \\
\text { utilization }\end{array}$ & Overhead \\
\hline $\begin{array}{c}\text { (OLB + } \\
\text { LBMM) }\end{array}$ & $x$ & $\checkmark$ & $x$ & $x$ & $\checkmark$ & $x$ & $\checkmark$ \\
\hline $\begin{array}{c}\text { Genetic } \\
\text { Algorithm }\end{array}$ & $x$ & $x$ & $x$ & $x$ & $\checkmark$ & $\checkmark$ & $x$ \\
\hline $\begin{array}{c}\text { VectorDot } \\
\text { Stochastic } \\
\text { Hill } \\
\text { Climbing }\end{array}$ & $\checkmark$ & $\checkmark$ & $x$ & $x$ & $\checkmark$ & $\checkmark$ & $x$ \\
\hline \begin{tabular}{c} 
LBVS- H \\
\hline
\end{tabular} & $x$ & $x$ & $x$ & $x$ & $x$ & $\checkmark$ & $x$ \\
\hline $\begin{array}{c}\text { Ant colony } \\
\text { optimization } \\
\text { algorithm }\end{array}$ & $x$ & $x$ & $x$ & $x$ & $\checkmark$ & $\checkmark$ & $x$ \\
\hline $\begin{array}{c}\text { Compare } \\
\text { and Balance }\end{array}$ & $x$ & $\checkmark$ & $\checkmark$ & $x$ & $\checkmark$ & $x$ & $x$ \\
\hline $\begin{array}{c}\text { Honey Bee } \\
\text { Foraging }\end{array}$ & $x$ & $x$ & $x$ & $x$ & $x$ & $\checkmark$ & $x$ \\
\hline
\end{tabular}

\section{References}

[1] G. Xu, J. Pang and X. Fu, "A load balancing model based on cloud partitioning for the public cloud", IEEE transactions on cloud computing year, (2013).

[2] Z. Chaczko, V. Mahadevan, S. Aslanzadeh and C. Mcdermid, "Availability and load balancing in cloud computing", international conference on computer and software modeling, (2011).

[3] S. Ray and A. de Sarkar, "Execution analysis of load balancing algorithms in cloud computing environment", International journal on cloud computing: services and architecture.

[4] A. Hans and S. Kalra, "Comparative study of different cloud computing load balancing techniques", Medical Imaging, m-Health and Emerging Communication Systems (MedCom), International Conference on IEEE, (2014).

[5] N. J. Kansal and I. Chana, "existing load balancing techniques in cloud computing: a systematic review", journal of information systems and communication. 
[6] Z. Chaczko, V. Mahadevan, S. Aslanzadeh and C. Mcdermid, "Availability and load balancing in cloud computing", International conference on computer and software modeling, (2011).

[7] K. Dasguptaa, B. Mandalb, P. Duttac, J. K. Mondald and S. Dame, "A genetic algorithm (ga) based load balancing strategy for cloud computing", International conference on computational intelligence: modeling techniques and applications (cimta), (2013).

[8] A. Singh, M. Korupolu and D. Mohapatra, "ACM/IEEE conference on Supercomputing, (2008).

[9] R. P. Padhy and P. G. P. Rao, "load balancing in cloud computing systems" thesis from national institute of technology", Rourkela-769 008, Orissa, India, (2011) May.

[10] S. Wang, K. Yan, W. Liao and S. Wang, 3rd International Conference on Computer Science and Information Technology, (2010), pp. 108-113.

[11] H. Liu, S. Liu, X. Meng, C. Yang and Y. Zhang, International Conference on Service Sciences (ICSS), (2010), pp. 257-262.

[12] A. Agarwal and S. Jain, "Efficient optimal algorithm of task scheduling in cloud computing environment", International journal of computer trends and technology (IJCTT).

[13] Y. Zhao and W. Huang, 5th International Joint Conference on INC, IMS and IDC, (2009), pp. 170-175.

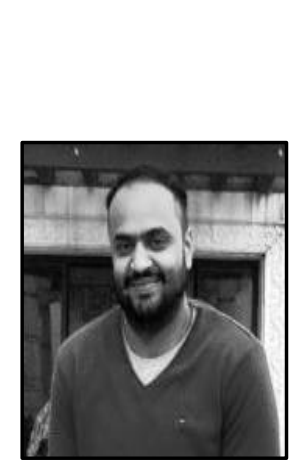

\section{Author}

Abhinav Hans, was born on 15-09-1990. He completed his B.Tech in Computer science and engineering from Lovely Professional University, Phagwara, Punjab, India in the year of 2013. $\mathrm{He}$ is persuing in M.Tech in Computer Science and Engineering from Guru Nanak Dev University, RC, Jalandhar. His field of interest are cloud computing, biomedical, big data, wireless sensor network, body area network. Till now he possess with various number of publications out of which much of them are in IEEEXPLORE and Journals. 
International Journal of Grid Distribution Computing

Vol.8, No.2 (2015) 